\title{
Effect of parametric design on landscape
}

\author{
Huang Geng ${ }^{1, a}$, Feng Xinqun ${ }^{2, b}$ Lin Feng $^{3, c^{*}}$ \\ 1,2,3No.1882 Yan'an Road,Chang ning District.200051 Shanghai, China \\ a hw_1209@126.com, b451496289@qq.com, ${ }^{\circ} 591769517 @ q q . c o m$
}

Keywords: parametric, landscape morphology, logic, system

Abstract: The nature, technology, human being determine the landscape morphology in the landscape design and construction process. Parametric is an emerging technical tool and thought. As a medium, it could build an overall relationship among natural site factors, functions and artificial construction, to control and visualize the process and result. Provision of adaptability to the environment is a fundamental parametric principle. With parametric thinking, final landscape morphology will reflect deeper landscape infrastructure, process and structure.

\section{Introduction}

In the history of China, a theory that man is an integral part of nature has influenced the city pattern and natural landscape for thousands of years, urban landscape morphology built along mountains, waters and terrains integrates the nature, architecture and human beings. the "Scenic City" put forward by Qian Xuesen just verified such view of nature. With the expansion of city size and human construction activities, landscape morphology is broken into pieces, landscape becomes supplementary to construction joints, becomes patches of the city. The proposal of landscape urbanism and ecological urbanism pulls landscape morphology back to a context of holistic view, landscape morphology becomes dominant in the connection of architectures. A landscape professor of Harvard University, Charles, gives an interpretation to landscape: landscape concerns more to build city texture, strengthens the link between human being and the city in a better way.

With the update of landscape concept, a new computer technology also penetrates into landscape design and has been developed rapidly. These designs may be described using such words as irregular, nonstandard, random, dynamic and so on. Such landscape morphology is the analysis result of various factors in the landscape site, a symbol of design complexity, such morphology of landscape is closely related to computer technology, enables landscape back to holistic level. Tool and form are paired, we need new tools and language forms to build new landscape morphology in a new era. Parametric design based on data analysis explains the relationship between site and final morphology as well as the generation process in a more scientific way, the application of parametric thinking method into new landscape design will definitely be of great significance for the construction of new type landscape morphology.

\section{Parametric theory and technology}

Theoretical basis of parametric design is based on Chaos Theory, whose core viewpoint is infinite natural world morphology through self-organization. Derivative change relationship is established between biological prototype change and morphological structure. Sinc the late 1990s, under the promotion of AA School of Architecture, nonlinearity and complexity science has been applied in architecture and design field taking parametric design as a medium. In the landscape field, it means 
the inclusion of all landscape factors in this organization from deep structure of the site to surface morphology of the site via a computer platform. The change of a minor environmental factor on site might influence overall change of a landscape, so overall dynamic system control of the landscape may be realized morphologically. The parametric design was born to solve comprehensive problems that could not be solved by traditional design methods, for example, the relationship among complexity, diversity of the site to be designed and the construction technology.

Parametric is an advanced computer model generation technology. Complex system and nonlinear science opened a new era since the 1960s, so people viewed correlated systems, as well as non-linear, irregular, irreversible, dynamic phenomena in the world. It was firstly used in industrial design, then extended to building field. With the progress of software basis and processing technology, it has realized the linking between data and morphology. It relies on key processes, such as site data, rules and function import, software morphogenesis, processing and breakdown etc. Parametric requires certain software support, for example, typical analysis software Ecotect, style software Rhino, logic software Grasshopper. Parametric design also requires special algorithm, including voronoi, L-system, Minimal Surface, swarm intelligence algorithm, cellular automaton, genetic algorithm, recursive algorithm, 3D restrained diffusion aggregation algorithm, multi-agent system etc. Now with the promotion of Building Information Modeling (BIM), parametric platforms Revit, Digital Project are also used in landscape design gradually. These software and technologies provide technical basis for visual morphology linking of parametric and landscape. (Fig. 1, 2) Architectural Association School of Architecture promotes technical progress and teaching practice of parametric design.
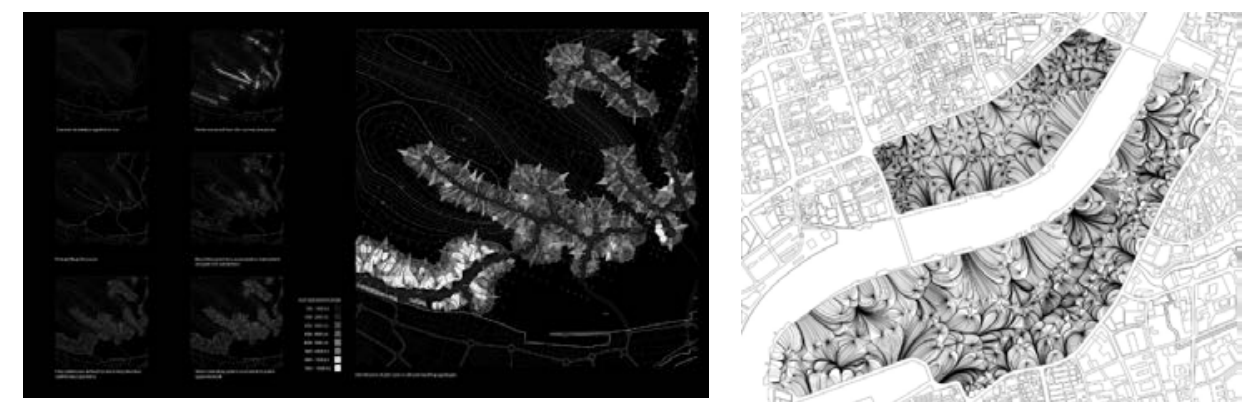

(Fig. 1, 2, Parametric Research \& Teaching of Architectural Association School of Architecture)

\section{Intervention of parametric in landscape morphology}

Intervention modes of parametric design may be divided into auxiliary parametric design and parametric design, the former assists design as a tool, the latter is a method, emphasizes the dynamic process between process variable and result morphology.

Thanks to technical progress, designers may use many parametric software tools to develop landscape morphology with parametric logic aesthetic characteristics for construction. (Fig. 3, 4) for example, Henderson Waves, in order to integrate the bridge with the environment, bridge morphology symbols 9 waves, the largest wave is $57 \mathrm{~m}$ long and $6 \mathrm{~m}$ high, bridge body adopts a tropical Yellow Balau hard wood, IJP Company completed the complicated hyperboloid structure using parametric technology, provided a dynamic graceful landscape place for pedestrians and

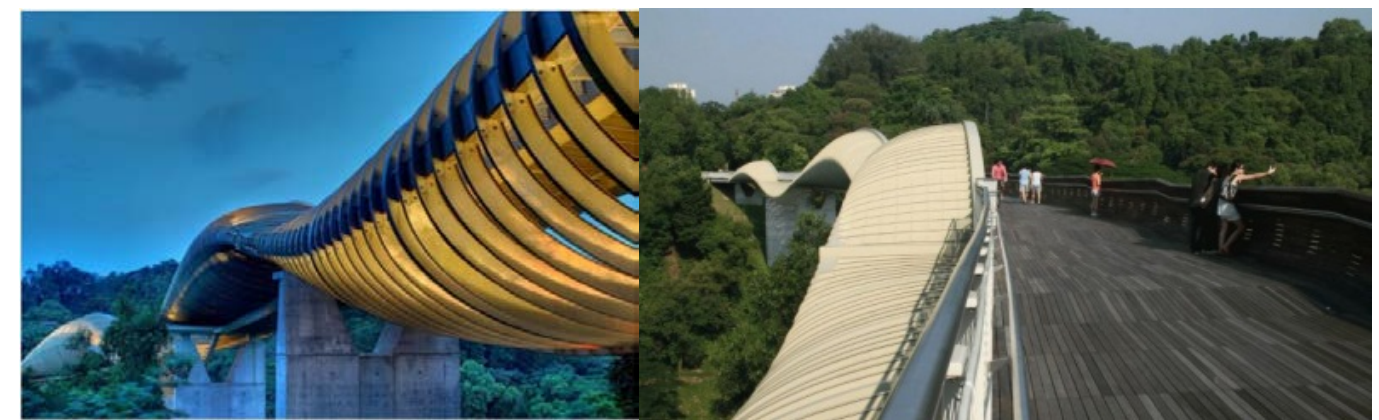


bicycles.

(Fig. 3, 4, Henderson Waves Footbridge)

Targeted parametric application containing parametric thought. Stress is laid on parameter prototype and logical relationship variables. For example, (Fig. 5, 6) canopy of AA School of Architecture designed by Feng Shuai, the morphology obtained from full utilization of rainwater fluid analysis.

(Fig. 5, 6, the Canopy Designed by Feng Shuai)

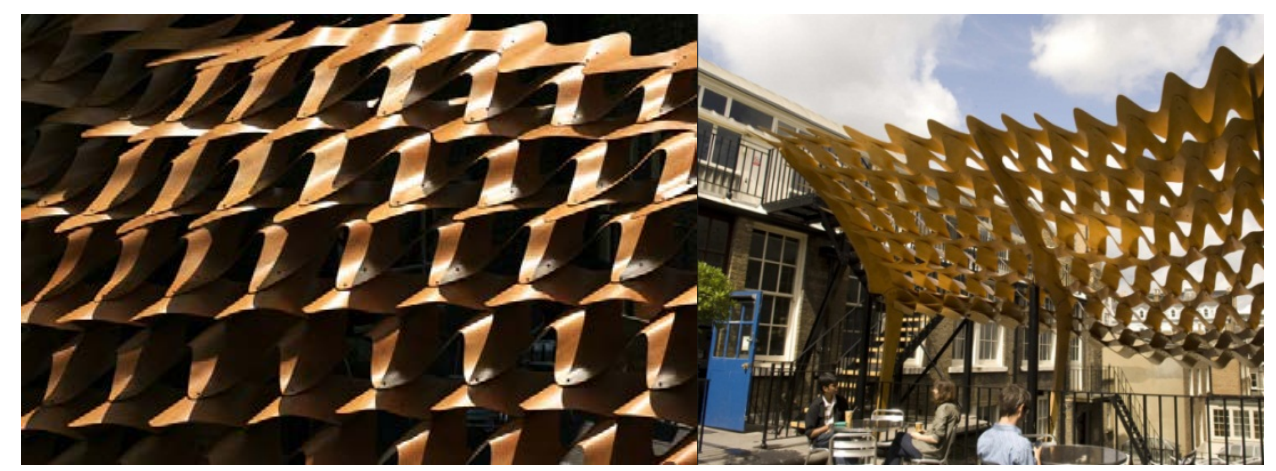

3.2 The difference between parametric and traditional design methods

The biggest difference between "parametric" planning design method and traditional method lies in the difference in their processes. Traditional method analyzes site conditions and problems, plots sketches, build models in computers, and then makes changes for better expression of final model. Such method from top to bottom as a whole targets final result and the design process intends to clarify and finalize initial fuzzy result. "parametric" working method emphasizes system and logic, design work passes through logic inference, starts from site conditions, finds out the relationship between influencing factors on site, formulates new "rules", then "generates" result, this is a design process from bottom to top. Such thinking mode is the precondition of "parametric" planning design.

Clear morphology data are unavailable for landscape site, so we set up basic site data information gradually based on the analysis of factors in site, the water, terrain, wind, sunshine comprise a site data system step by step. Function determines form, then site function is attached to the site data base, building function, pedestrian flow function, ecological function etc. re integrated to the entire site morphology gradually. Similarly, parametric inevitably involves specific problems, for example, specific work flow, operation mode, decision-making and process of thinking etc.

Parametric technology becomes mature in building field, exhibits its advantages in landscape design preliminarily, especially for creating more flexible, dynamic, smooth morphology.

I. Digital technology is used to expand natural organic morphology. In the landscape design, in order to create more creative morphology, digital software is introduced to create dynamic natural morphologies more conveniently in virtue of interference, particle, fractal methods.

II. Parametric design integrates complex systems. Now that landscape of new morphology depends on numerous external and inherent influences. We regard comprehensive action of the factors that influence landscape as a complex system, factors in this system may be called variables, if we study the landscape site and functions, find out the rules among these environmental factors, then we could utilize computer technology to generate site morphology, space and structure, and may obtain solutions for the site by changing the value of variables. According to such operating method, as a result, landscape morphology may also generate flowing, irregular sense of site, parametric technology may solve site complexity more effectively.

III. Parametric generation and analysis process will increase rationality of the design. Parametric method is based on objective law of the nature itself. It extracts critical factors of the nature to build a three-dimensional dynamic model, so is more scientific and reasonable. Morphology correlation 
IV. Parametric design offers huge and appropriate data correlation processing capacity for landscape. Adaptability is the principle of parametric method.

$\mathrm{V}$. Parametric generation mode is affected by non-linear science, the design result broken down automatically by specific software, and low technology level is shown in construction breakdown, processing and installation.

\section{Parametric ecological integration for multi-dimensional landscape morphology}

Landscape is an entity with life. It consists of terrain, soil, water body, vegetation, human activities and other factors. These factors develop dynamic balance through competition, restriction and synergy. Site is the foundation of landscape, site factors include rainwater, sunshine, wind, plant, river etc. The first step to control landscape morphology is site datamation, datamation reveals the relationship among site elements, so it has obvious local morphology and characteristic. Data collection and indexation is always the starting point to interpret site. Based on urbanism landscape, parametric design extends to landscape design while dominant in architectural morphology. (Fig. 7, 8, Analysis of PLASMA STUDIO on Site Datamation Generation)
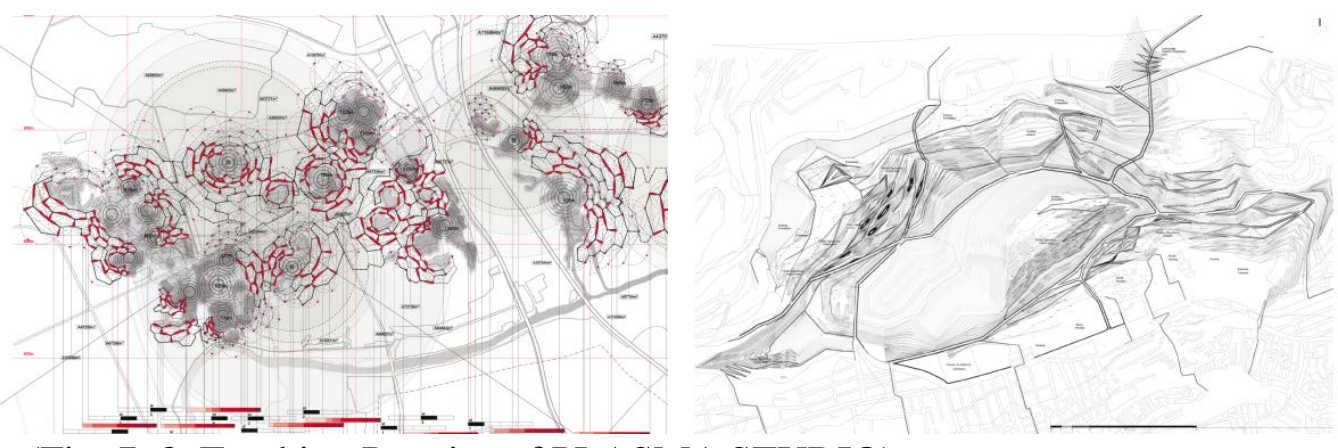

(Fig. 7, 8, Teaching Practice of PLASMA STUDIO)

At the early stage of landscape design, designer will face two problems, firstly, recognize current status, then determine the design method. Parametric design practice undoubtedly starts from method, and regresses to the site to be designed. The body generated by this technology finally returns to the level corresponding to human behavior and experience. For example, (Fig. 9, 10), Motril footbridge designed by the firm Guallart Architects, connects Explanadas garden and Pueblos de las Américas parking lot where height difference exists. Bridge structure and style adopt the same style element, fully adapt to ambient massif and environment, facilitate people including the disabled to use. In order to ensure smooth traffic in the streets beneath the bridge, minimum distance between streets in main bridge body is $5 \mathrm{~m}$. Steel sheets of different thickness are cut and folded to form continuous style element. LED lights are hidden ingeniously to illuminate the bridge and road. Designer realizes the combined design of this function and morphology through parametric design and script editing.
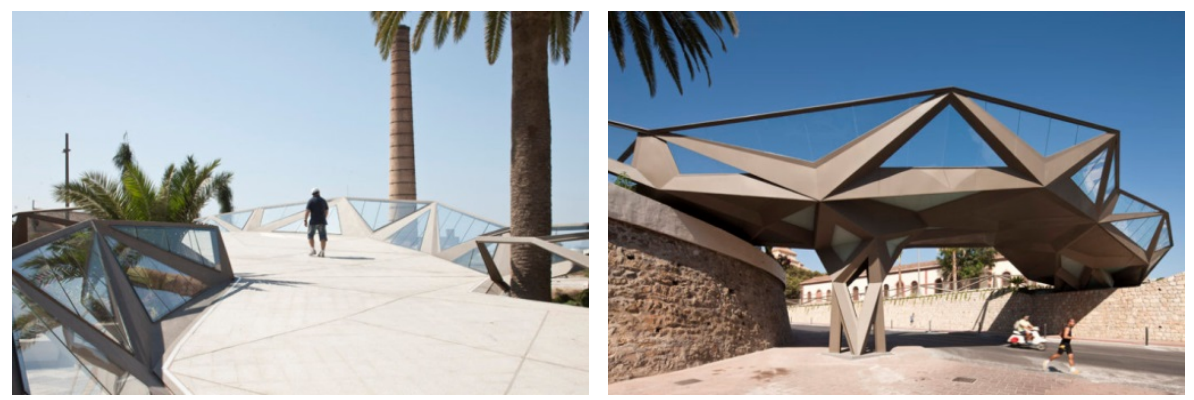

(Fig. 9, 10, Motril Footbridge)

The key of landscape urbanism is the design according to continuous landscape morphology, 
rather than isolated architectural form, the integration of utilities, buildings, roads, greening into
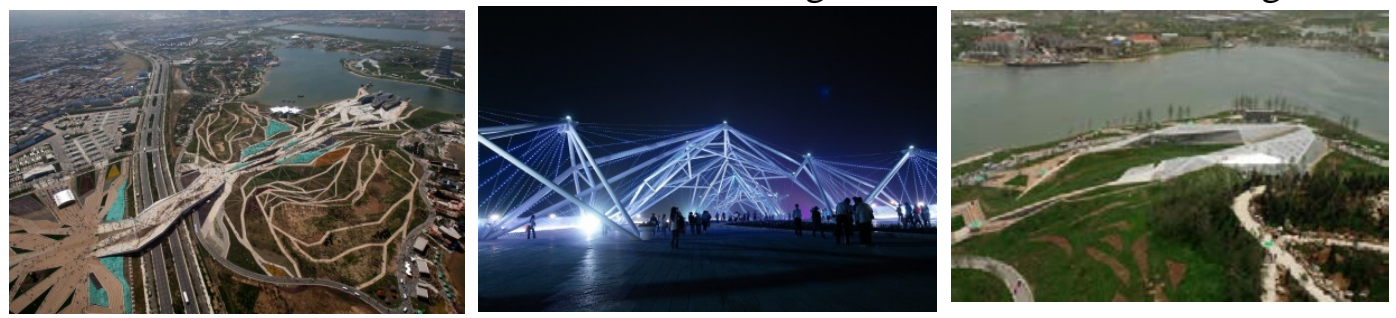

systematic landscape morphology using parametric technology. (Fig.11, 12, 13) Xi'an World Horticulture Expo planning presided by PLASMA from UK is an integration case from building to landscape morphology. This landscape scheme named "Flowing Garden" is established on the basis of flow and relevance, digital, visualization is realized on the basis of grid system to express landscape site relationship, so as to form the frame of the entire garden, then deformation is performed on the grid according to the fluctuant massif around the site. Through analysis, rhombus diagonal in the grid system serves as walkway, different road levels are assigned, and corresponding structures are extended to the rest sites. Triangular zones in the grid system are plant growth zone and wetland, similar to the patches in ecosystem. Chief designer Eva Castro said, “'flowing garden' is an organic organization system, which contains an information set of different levels based on surrounding environment. Any building in the grid system changes in this network structure, and develops its final morphology. Landscape and building become very fuzzy. Flowing is highlight of the entire scheme, building body and landscape construction adopt the same logic in form, at the same time, for geometric setting, building gathers landscape energy, landscape extends the building horizontally, overall morphology is holistic and meets functional requirements. "flowing garden" does not utilize any specific parametric software, design emphasis is the utilization of "parametric concept" or "parametric thinking" in landscape system construction process. This project involves overall integration of landscape and building based on parametric thought.

(Fig.11, 12, 13, Xi'an World Horticulture Expo Landscape designed by PLASMA STUDIO)

\section{Conclusion}

parametric method is undoubtedly a tool and concept with advantages. software may generate various unique bodies according to the parameter conditions. However, huge risk also exists in the application of this technology. If we only look for visual aesthetics and impactful style, formism result will be obtained. Parametric emphasizes derivative relationship between site factors and morphology from the start of the design, sets up a visible correlation between the process and result. Such technical innovation, may bring about new concept, method, process and result for landscape design. Parametric thinking goes far beyond parametric technology, and even without parametric software, more possibilities will be given to the design. Parametric generation basis is the logic of natural science, parametric method is relatively objective and scientific. However, landscape morphology is only presentation, while parametric thinking may link the presentation with landscape structure and ecology at a deeper level, with the extension of landscape system concept, landscape will become a connector among building, environment and human being, and will become more complicated and diversified from function to morphology. Parametric application just correlates these complex systems. More designers are required to participate in the practice of parametric landscape morphology in the future.

\section{References:}

[1] Tom Verebes. New Computational Paradigms in Architecture. Beijing: Tsinghua University 
Press., 2012

[2] Chi Zhiwei, Chen Jie, Zhang Deshun. Application Progress of Parametric Design and Its Enlightenment On Landscape Design (Process in Application of Parametric Design and Its Inspiration to Landscape Design). Beijing: Chinese Landscape Architecture, 2012

[3] Shen Kang. Parametrical Design and Locality. Guangzhou: Southern Architecture, 2010

[4] Kuang Wei. Overview of Current Development Status of "Parametric" Planning Design for Landscape Architecture and Thinkings. Landscape Architecture, 2013

[5] Huang Weixin, Xu Weiguo. Parametric and Generative Landscape Design). Beijing: Landscape Architecture, 2013

[6] Xu Weiguo. Digital Tectonics [J]. Architectural Journal, 2009(1): 61-68. 